\title{
Frictionless Surface Gravity Waves
}

\section{Kern E. Kenyon}

4632 North Lane, Del Mar, USA

Correspondence to: Kern E. Kenyon, kernken@aol.com

Keywords: Surface Gravity Waves, No Friction

Received: March 7, $2020 \quad$ Accepted: March 29, 2020

Published: April 1, 2020

Copyright $\odot 2020$ by author(s) and Scientific Research Publishing Inc.

This work is licensed under the Creative Commons Attribution International License (CC BY 4.0).

http://creativecommons.org/licenses/by/4.0/

\section{Open Access}

\section{ABSTRACT}

A physical explanation is given for the observations that ocean surface gravity waves can travel up to half way around the world from generation in a wind storm to dissipation on shore. Inherent in these waves is an orbital fluid particle motion, known from laboratory experiments, that has no friction according to the Navier-Stokes equations. The prediction is based on application of Bernoulli's law to all the closed orbital paths of the fluid particles and the cross-stream force balance on the particles between a pressure gradient and the centrifugal force in each orbital loop.

\section{INTRODUCTION}

In the title is an idea that was given one sentence earlier [1], therefore a more detailed explanation is presented here. Classically the theory of the surface gravity wave cannot explain the absence of friction because of the foundational mathematical assumption: irrotational motion with zero friction built into it. This restricts the waves to having no friction from the start, regardless of what theoretical constructions come next.

Ocean recordings of surface gravity waves have long amazed scientists and engineers by the great distances they can travel from the storms that created them. In a few striking cases an instrument near Yakutat, Alaska, together with several other instruments (6 in total) spaced out along a great circle route in the open water of the North Pacific, obtained signals whose inferred source was a storm in the Indian Ocean, halfway around the world from Yakutat [2]! Certainly these waves had to have passed through at least one wind belt along the way, which supposedly might have caused them a disturbance if not total destruction. Although surprise was registered concerning these extreme propagation distances, no reason was offered at that time for how they could have taken place.

Underneath the wave surface there are always fluid velocities everywhere in the zone of wave influence, and velocity gradients as well. Why don't these waves just grind to a halt after only traveling a couple of wavelengths, with friction providing the grinding?

\section{ARGUMENT}

Circular orbits of all the fluid particles during the translation of surface gravity waves is an essential 
feature which does not come from any theory. Observations of the movement of small neutrally buoyant particles are the best way to illustrate the circular orbital paths. No other type of wave in physics has such a property, and it implies that surface gravity waves not only possess orbital angular momentum of the fluid particles, but they propagate it along with energy and linear momentum.

Two examples of streak photographs in a laboratory setting $[3,4]$, showing neutrally buoyant particles underneath surface gravity waves, are instructive to view. In both cases there are many closed streamline loops distributed throughout the water column.

Select any one closed streamline (loop) and apply Bernoulli's law to it.

$$
p=\text { const }-\frac{1}{2} \rho v^{2}
$$

where $p$ is the pressure, $v$ the velocity tangent to the streamline and $\rho$ is the constant density. This application has been done before but in a different context [5]. Now form the cross-stream force balance for any fluid particle in the loop (e.g. [6])

$$
\frac{\mathrm{d} p}{\mathrm{~d} r}=\rho \frac{v^{2}}{r}
$$

where $r$ is the radial distance from the center of the loop.

Equations (1) and (2) are two equations in two unknowns, pressure and velocity. They are both non-linear. Eliminate the pressure from them to get one equation in one unknown, velocity

$$
\frac{\mathrm{d} v}{\mathrm{~d} r}=-\frac{v}{r}
$$

which is linear and has the solution

$$
v=\frac{\text { const }}{r}
$$

There is no friction associated with Equation (4), according to the Navier-Stokes equations in polar coordinates. Since (3) is linear, it can be applied to other loops just as well, and the total solution will be the sum of all the individual ones.

All fluid particles under the wave surface undergo an orbital motion, not just those illustrated in the streak photographs. In summary, the argument leads to the conclusion that the surface gravity wave is frictionless.

\section{DISCUSSION}

Limitations of the irrotational theory for describing the surface gravity wave are gradually becoming known, and the present essay points in the same direction, illuminating another limitation or two. There has never been a comprehensive attempt to compare theory and observations, but consider by itself the logic of the theoretical starting point in most hydrodynamics text books that treat such waves [5], which is Laplace's equation and the velocity potential $\varphi$.

$$
\varphi=f(z) \sin (k x-\omega t)
$$

As usual, from the velocity potential function in Equation (5) the fluid velocity is computed by taking derivatives of it. Standing alone what this equation means then is that the fluid itself is translating! That makes no sense. Only the shape of the surface propagates.

If the main result expressed above, frictionless surface gravity waves, holds up, then it fits in with a philosophy described twice before in connection with the hurricane and the tornado: during fluid flows seen persisting in nature they have the characteristic of involving the least amount of friction among all those possibilities that could take place. Zero friction would be the optimum of course. 


\section{CONCLUSION}

From the observed orbital motion of fluid particles in a propagating surface gravity wave, physical reasoning is applied which leads to the prediction that the wave is inherently free of friction based on the Navier-Stokes equations. Thus a way is opened up to understand qualitatively how ocean surface gravity waves can travel up to half way around the world, which has been known to occur over the past couple of centuries.

\section{CONFLICTS OF INTEREST}

The author declares no conflicts of interest regarding the publication of this paper.

\section{REFERENCES}

1. Kenyon, K.E. (2019) Bernoulli Loops with no Friction. European International Journal of Science and Technology, 8, 25-28.

2. Snodgrass, F.E., Groves, G.W., Hasselmann, K.F., Mller, G.R., Munk, W.H. and Powers, W.H. (1966) Propagation of Ocean Swell across the Pacific. Philosophical Transactions of the Royal Society of London, 259, 432-437.

3. Sommerfeld, A. (1964) Mechanics of Deformable Bodies. Academic Press, New York, 181.

4. Weigel, R.L. (1964) Oceanographical Engineering. Prentice-Hall, New Jersey, 19.

5. Lamb, H. (1945) Hydrodynmics. 6th Edition, Dover Publications, New York, 365.

6. Batchelor, G.K. (1967) An Introduction to Fluid Dynamics. Cambridge University Press, San Diego, 203. 